\title{
Malignant Soft Tissue Sarcoma at the Site of Corticosteroid Injection in a Dog
}

\author{
Aulakh $\mathrm{KS}^{*}$, Kielb JL ${ }^{2}$, Hsiao $\mathrm{SH}^{3}$, Barnes $\mathrm{K}^{1}$ and Aulakh $\mathrm{HK}^{1}$ \\ ${ }^{1}$ Department of Veterinary Clinical Sciences, School of Veterinary Medicine, Louisiana State University, Skip Bert- \\ man Drive, Baton Rouge, LA 70803, USA \\ ${ }^{2}$ Department of Veterinary Clinical Medicine, College of Veterinary Medicine, University of Illinois at Urbana- \\ Champaign, 2001 S. Lincoln Ave. Urbana, IL 61802, USA \\ ${ }^{3}$ Veterinary Diagnostic Laboratory, College of Veterinary Medicine, University of Illinois at Urbana-Champaign, \\ 2001 S. Lincoln Ave Urbana, IL 61802, USA
}

*Corresponding author: Aulakh KS, BVSc \& AH, MS, MS, DACVS-SA, Department of Veterinary Clinical Sciences, School of Veterinary Medicine, Louisiana State University, Skip Bertman Drive, Baton Rouge, LA 70803, USA, E-mail: ksaulakh@gmail.com

Citation: Aulakh KS, Kielb JL, Hsiao SH, Barnes K, Aulakh HK (2018) Malignant Soft Tissue Sarcoma at the Site of Corticosteroid Injection in a Dog. J Vet Sci Ani Husb 6(1): 102. doi: 10.15744/2348-9790.6.102

Received Date: February 17, 2016 Accepted Date: February 26, 2018 Published Date: February 28, 2018

\begin{abstract}
An 11-year-old spayed female dog was presented for a large mass on the left proximal pelvic limb. The mass appeared three months prior to presentation in the location of a previous corticosteroid injection, Kenalog ( $0.05 \mathrm{mg} / \mathrm{kg}$ triamcinolone, CAS\# 76-25-5). No vaccinations or injections had been previously given in this area. The mass spanned from the left stifle to the coxofemoral joint and was firm, circular, non-moveable, and diffusely mixed within the musculature of the thigh. A left external complete hemipelvectomy was performed. Histopathology was consistent with a Grade III fibromyxosarcoma. The dog died naturally at home five months after surgery. This is the first account of association of a corticosteroid injection with formation of a fibromyxosarcoma in a dog.
\end{abstract}

Keywords: Injection Site Sarcoma; Fibromyxosarcoma

\section{Case Description}

An 11 year old, $7.7 \mathrm{~kg}$, spayed female Wire Fox Terrier was presented for evaluation of a 3-month history of a very large mass on the left pelvic limb. The dog had a history of suspected skin allergies that began when she was 9 years old. No testing or skin biopsies were performed to confirm this, but she appeared to respond to empirical treatment for atopic dermatitis. She was treated with subcutaneous Kenalog $(0.05 \mathrm{mg} / \mathrm{kg}$ triamcinolone $)$ injections in the dorsal cervical region every four weeks for the past two years. Three months prior to surgical evaluation, a $0.05 \mathrm{mg} / \mathrm{kg}$ Kenalog injection was given subcutaneously in the left pelvic limb. Two days after the injection, a small firm mass appeared in the region of the injection site. The dog had no previous history of having an injection or vaccination in the left pelvic limb. She was presented to her regular veterinarian for evaluation of the mass, which had grown rapidly in the two weeks post-injection and was referred to another hospital. Fine needle aspiration was performed and showed a large amount of blood and some spindle shaped/mesenchymal cells. Because this sample was inconclusive, surgical biopsy was recommended for definitive diagnosis. At the time of incisional biopsy, the mass was softball size. Three view thoracic radiographs were within normal limits and there was no evidence of metastasis. Histopathology revealed a mass composed of plump haphazardly arranged spindle to stellate-shaped cells. The cells had moderate nuclear size, greater than 20 mitotic figures seen in 10 high power fields, and adipose tissue infiltrates. Radical resection of the mass and referral to a veterinary surgeon was recommended. Due to financial constraints, the owner opted not to follow through with consultation and surgical removal at that time.

The dog was presented to us for further evaluation and treatment 8 weeks after incisional biopsy. On presentation, the dog was bright, alert, and responsive with normal heart and respiratory rates. No peripheral lymphadenopathy was palpated. The dog had a non-weight bearing lameness of the left hind limb. Over the left lateral femur, there was a $10 \mathrm{~cm}$ x14 cm x13 cm, firm, circular, non-moveable mass that was diffusely mixed within the musculature of the thigh (Figure 1). It spanned from the coxofemoral joint to the stifle. The mass appeared red on the lateral aspect and spanned medially. On rectal palpation, the mass extended to the ventral midline. It was not painful on palpation, but was warm to the touch. Three-view thoracic radiographs were repeated and there was no evidence of metastasis. An abdominal ultrasound, complete blood count and chemistry were within normal limits. 


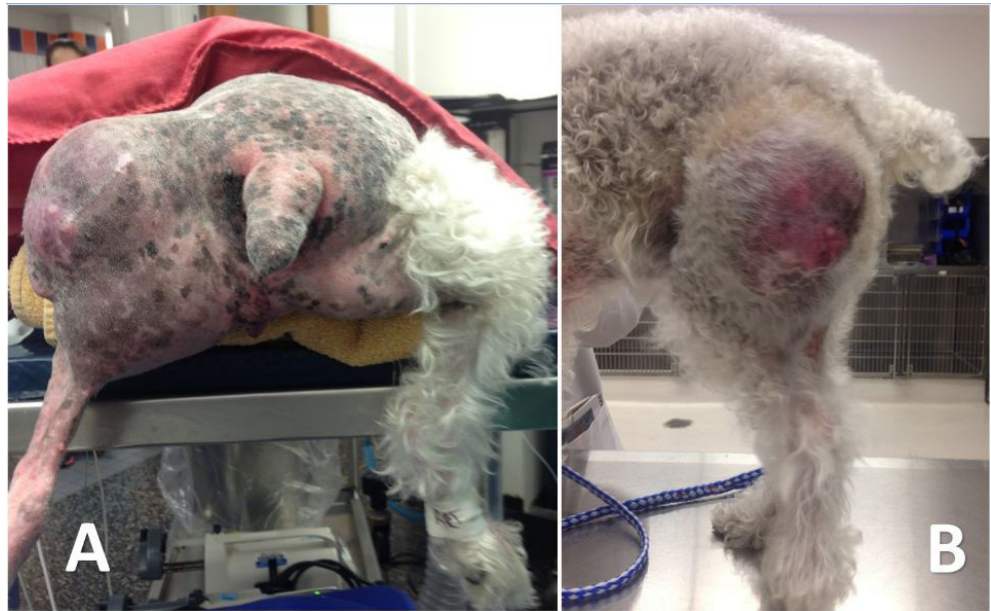

Figure 1: Photographs of the mass on the left pelvic limb of an 11 year-old spayed female Wire Fox Terrier; (A) Caudocranial and (B) Lateral view of the mass which extended distally from the coxofemoral joint to the stifle and spanned from the lateral stifle to midline

A Computed Tomographic (CT; Lightspeed 16, GE Healthcare, Waukesha, WI, $0.625 \mathrm{~mm}$ bone algorithm, $2.5 \mathrm{~mm}$ standard algorithm) scan of the left hind limb and pelvis revealed a large, rounded, well-marginated, mildly contrast enhancing, soft tissue attenuating mass completely distorting the normal musculature of the left proximal pelvic limb (Figure 2). The mass extended from the caudal stifle area to the pelvis measuring approximately $10 \mathrm{~cm}$ in length, $14 \mathrm{~cm}$ in height, and $13 \mathrm{~cm}$ in depth. The mass displaced the soft tissue ventral to the pubis, but did not appear to cross midline. The proximal aspect of the mass crossed dorsal to the left coxofemoral joint and just caudal to the ilium before tracking ventrally to the lumbosacral junction and to the left L6-7 intervertebral foramen. As the mass followed this path, it did enter the left side of the pelvic canal, resulting in moderate right-sided displacement of the terminal colon. Based on the imaging, it was suspected the dog had a locally aggressive soft tissue tumor, which required amputation. Based on the invasive nature of the mass on CT and the previous histopathology results from incisional biopsy, a radical surgical excision with a left external complete hemipelvectomy was recommended to provide best chance for complete resection of the invasive mass with clean margins. A urinary catheter was placed pre-operatively for easy identification of the urethra during surgery and for post-operative care. The left pelvis and hind limb with associated musculature were amputated. The transverse process of L7 on the left side was removed to allow transection of the L7 nerve root at the level of the vertebral foramen to try to achieve complete margins. The abdominal and pelvic cavities were closed using the abdominal musculature, internal obturator and epaxial muscles. Due to the large amount of dead space with a massive resection, a Jackson-Pratt drain was placed in the surgical site to allow for adequate post-operative drainage. The dog recovered uneventfully post-operatively.

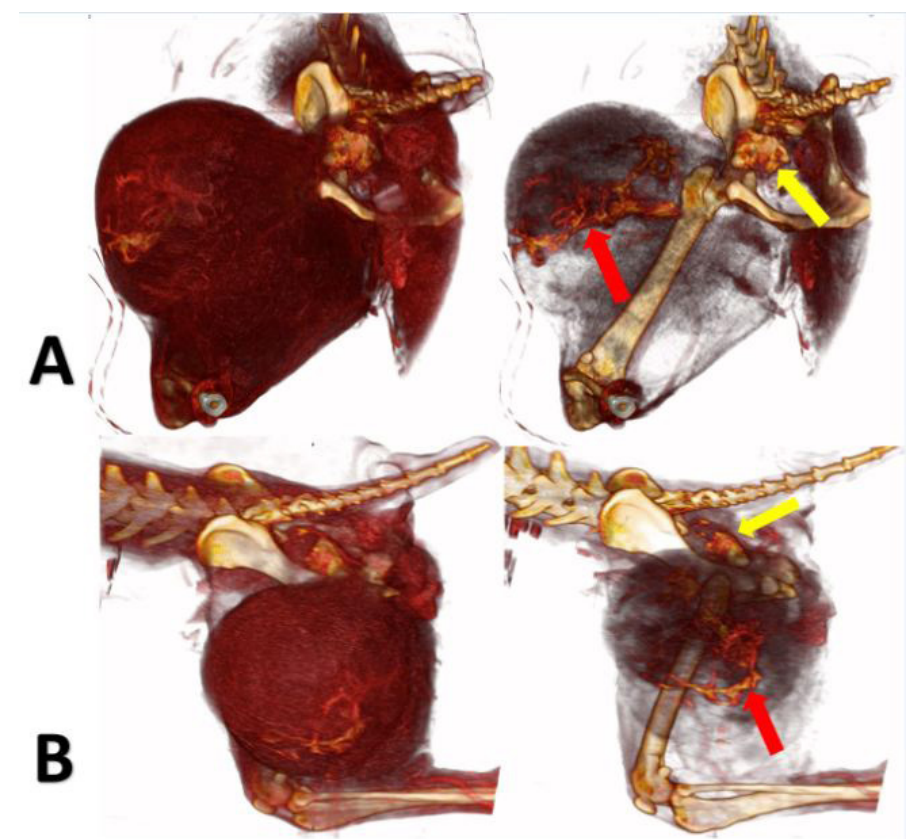

Figure 2: Three dimensional volume-rendered computed tomographic images of the mass on the left pelvic limb of the dog; (A) Caudo-cranial view; (B) Lateral view. Images on the left are volume rendered such that more soft tissue structures are visible. Images on the right are volume rendered such that more skeletal structures are visible. Amorphous mineralized material within the mass (red arrows) is better visualized in images on the right. The proximal aspect of the mass crosses dorsal to the left coxofemoral joint and just caudal to the ilium (yellow arrows) 
Gross examination of the amputated left rear limb confirmed a large, 11.5 X 10.5 X $9.5 \mathrm{~cm}$, soft tissue mass at the caudal-lateral aspect of the femur. The mass was multi-nodular, partially encapsulated yet invasive, white to tan, solid but soft, and bulged on the cut surfaces (Figure 3A). It was firmly adhered to the caudal aspect of the stifle joint capsule, but was easily dissected from the femur and pelvis. The surgical margin was inked, and the mass appeared to be completely excised with a greater than $2 \mathrm{~mm}$ barrier along the closest margin at the caudal-medial aspect of the mass. Additionally, a second small, 3 X 2 X $2 \mathrm{~cm}$, bony mass was also discovered at the ventral-medial aspect of the left pelvis adjacent to the body of the ilium. The small mass was easily separated from the pelvic bone, and was not associated with the first large mass. Histopathology examination revealed that the large mass comprised of erratic streams of neoplastic fusiform cells with various degrees of differentiation. In relatively differentiated regions, the neoplastic cells were relatively small and elongated with a small amount of eosinophilic, fibrillar cytoplasm, and abundant intercellular collagenous and myxoid matrix (Figure 3B). Nuclei were small, oval and hyperchromatic with stippled chromatin, indistinct nucleoli, and rare mitosis. In poorly differentiated regions, the neoplastic cells were larger with a small amount of eosinophilic, fibrillar cytoplasm, and scant intercellular collagenous matrix (Figure 3C). Nuclei were abnormally large with vesicular chromatin and prominent nucleoli. There was marked anisocytosis, marked anisokaryosis, and a high mitotic index (36 mitotic figures per 10 active 400X fields). Up to $40 \%$ of tumor parenchyma was necrotic in the poorly differentiated regions. No evidence of tumor metastasis was observed in the popliteal lymph node. Complete excision was achieved with a greater than $2 \mathrm{~mm}$ of muscular barrier or a greater than $10 \mathrm{~mm}$ of fatty barrier from the margins of the specimen. Based on the characteristics of malignancy with abundance of intercellular collagenous and myxoid matrix, the tumor was diagnosed as a grade III, soft tissue sarcoma (fibromyxosarcoma). The neoplastic cells were immunohistopathologically negative for smooth-muscle specific actin. Although the small mass was also comprised of erratic streams of neoplastic fusiform cells, it contained embedded islands of osteoid spicules and osseous trabeculae (slide 3D). The neoplastic cells had a moderate amount of eosinophilic to amphophilic cytoplasm, and large, oval, euchromatic nuclei with stippled chromatin and prominent nucleoli. There was marked anisocytosis, moderate anisokaryosis, and a high mitotic index (98 mitotic figures per 10 active 400X fields). Microscopic diagnosis was an extra-skeletal osteosarcoma.

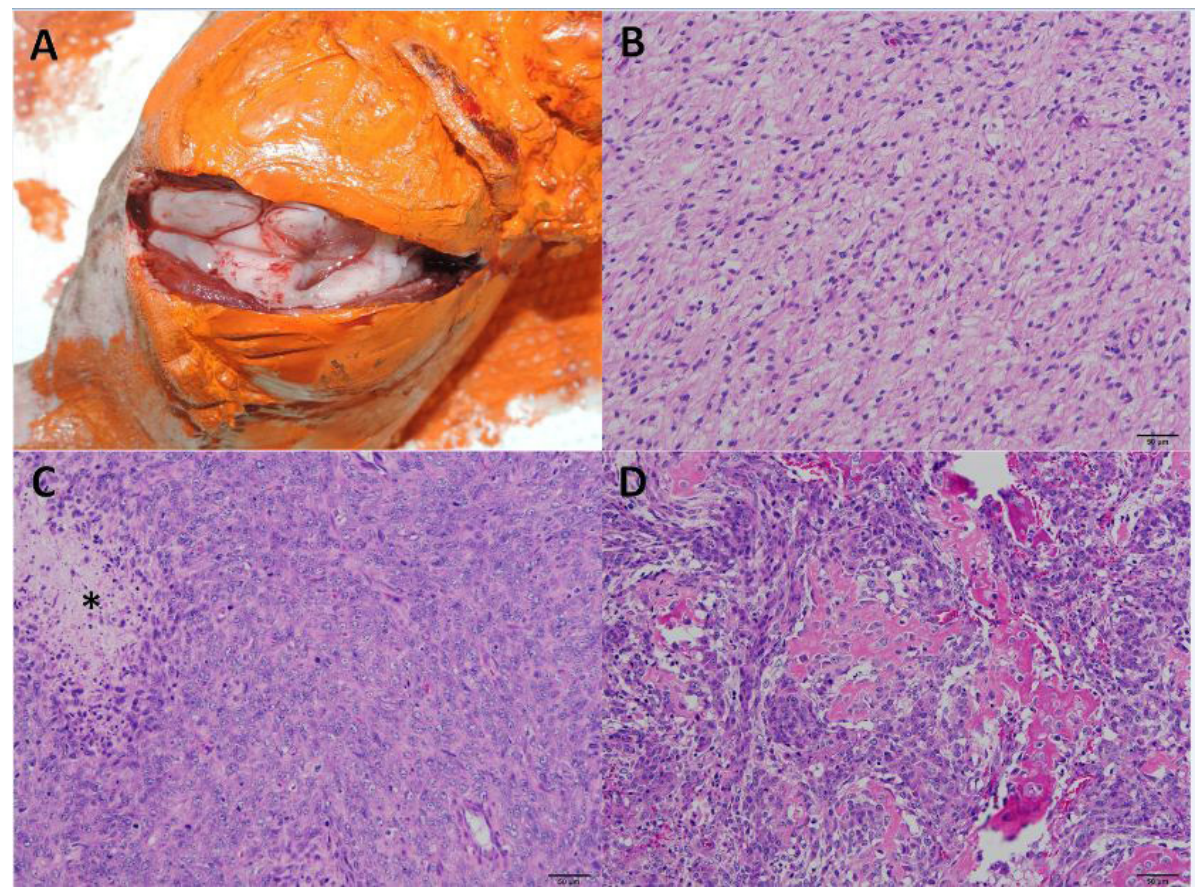

Figure 3: Gross and histopathology images of the fibromyxosarcoma (A-C) and an extra-skeletal osteosarcoma (D) in the left pelvic limb of an 11 year-old, spayed female Wire Fox Terrier; (A) A large soft tissue mass is present at the caudal-lateral aspect of the left femur. The mass is multi-nodular, partially encapsulated yet invasive, white to tan, solid but soft, and bulged on the cut surfaces. Surgical margin is inked; (B) In a relatively differentiated region of the mass, the neoplastic cells are relatively small and elongated with abundant intercellular collagenous and myxoid matrix. Nuclei are small and hyperchromatic with rare mitosis. H\&E stain. Bar = 50 micrometer; (C) In a poorly differentiated region of the mass, the neoplastic cells are larger with and frequent tumoral necrosis $\left(^{*}\right)$ and scant intercellular collagenous matrix. Nuclei are abnormally large with vesicular chromatin and a high mitotic index. Microscopic diagnosis was a grade III, soft tissue sarcoma (fibromyxosarcoma). H\&E stain. Bar = 50 micrometer; (D) A second small mass at the ventral-medial aspect of the left pelvis comprises of erratic streams of neoplastic fusiform cells with embedded islands of osteoid spicules and osseous trabeculae. The neoplastic cells have large, euchromatic nuclei with a high mitotic index. Microscopic diagnosis is an extra-skeletal osteosarcoma. H\&E stain. Bar $=$ 50 micrometer

The patient did well under general anesthesia and recovery was unremarkable. She was maintained on LRS intravenous (60ml/ $\mathrm{kg} /$ day) fluid therapy post-operatively. The pain was managed with lidocaine (Lidocaine, 30microgram $/ \mathrm{kg} / \mathrm{min}$ ), fentanyl (Fentanyl, 3microgram $/ \mathrm{kg} /$ hour) and ketamine (Ketamine, 2 microgram $/ \mathrm{kg} / \mathrm{min}$ ) intravenous constant rate infusions. Methadone (Methadone, $0.25 \mathrm{mg} / \mathrm{kg} \mathrm{IV)} \mathrm{was} \mathrm{administered} \mathrm{every} 4$ hours as needed for added pain control and acepromazine (Acepromazine, 
$0.01 \mathrm{mg} / \mathrm{kg}$ IV) was given for anxiety as needed. Twenty four hours post-operatively, she was weaned off the constant rate infusions and her pain was controlled with methadone IV $0.25 \mathrm{mg} / \mathrm{kg}$ every 4 hours. Due to her severe pyoderma, cefazolin (Cefazolin 22 $\mathrm{mg} / \mathrm{kg}$ IV q8 hours) was continued post-operatively. Once she was eating, she was transitioned to cephalexin (Cephalexin $33 \mathrm{mg} / \mathrm{kg}$ cephalexin PO q12 h) and continued for two weeks. The Jackson-Pratt drain was monitored for fluid output. The fluid production from drain was low therefore the drain was removed two days post-operatively. The patient was discharged from the hospital with two weeks of tramadol (Tramadol, 3.3mg/kg PO q 8hours), carprofen (Rimadyl, 2.2mg/kg PO q12 hours) and cephalexin (Cephalexin, 33mg/kg PO q12 hours).

The patient was doing well at the two week post-operative recheck examination. She was having some difficulty posturing to defecate, but the owner was very happy with her progress and quality of life. At this time, it was discussed with the owner that the best chance of curing this cancer, would be to perform metronomic chemotherapy. The owner was not interested in pursuing further treatment. At the time of follow-up via a telephone conversation, the owner reported that the dog was deceased five months after surgery. The owner reported that approximately one month after surgery she began experiencing urinary incontinence. After evaluation by a veterinarian, no cause was identified. The dog was then reported to become gradually weak in the right hind limb. She was unable to support herself while urinating and defecating. Five months after surgery, she died naturally at home.

\section{Discussion}

Soft tissue sarcomas are common tumor types that are of mesenchymal cell origin. These tumors are characteristically slow growing, locally invasive and have a low metastatic rate [1]. Soft tissue sarcomas are a broad category that can be further broken down to more specific tumors. Typically soft tissue sarcomas include: fibrosarcoma, myxosarcoma, hemangiopericytoma and peripheral nerve sheath tumor. Other sarcomas, such as rhabdomyosarcoma, liposarcoma, leiomyosarcoma, synovial cell sarcoma, malignant fibrous histiocytoma and undifferentiated sarcoma, may also be included in the list of soft tissue sarcoma. However, histiocytic sarcoma, hemangiosarcoma, osteosarcoma, malignant melanoma and oral sarcoma are excluded. Since this particular soft tissue mass in our case report expressed abundant intercellular collagenous/myxoid matrix and did not exhibit a cellular pattern or cytomorphology typically seen in hemangiopericytoma, peripheral nerve sheath tumor or other sarcomas, it was further classified as a fibromyxosarcoma [1,2]. Soft tissue sarcomas are graded from 1-3 based on level of differentiation, mitotic index and necrosis $[1,2]$.

Fibrosarcomas can be associated with injection site sarcomas (ISS) in cats and occasionally dogs [1-3]. There are some documented cases of dogs having ISS, which show similar histopathological characteristics to those seen in cats, such as, subcutaneous location, areas of necrosis, inflammatory response, and neoplastic mesenchymal cells [3]. Injection site sarcomas are known to be very fast growing, locally invasive and have high mitotic rates [1]. They are composed of elongated cells with a fibrovascular stroma. They often have an area of necrosis with the infiltration of lymphocytes, macrophages and multinucleate giant cells [1]. At this time, one etiology has not been determined for the cause of injection site sarcomas. Most commonly injection induced sarcomas appear after routine vaccination in cats [4]. It has been proposed that the adjuvant of the modified live virus is the cause of local inflammation and tumor growth, but with growing case reports and further studies being performed, this is not likely the sole cause [4]. Findings for the dog in this case report are worth documenting due to the potential correlation between the corticosteroid injection and the development of a soft tissue sarcoma. To our knowledge, this is the first report of a corticosteroid injection associated with a soft tissue sarcoma in a dog. In cats, long acting injectable steroids, such as methylprednisolone, dexamethasone and triamcinolone have been involved in the occurrence of injection site sarcomas [5]. No vaccines were administered to these reported cats, which lead to the conclusion that other injectable medications are capable of tumorigenesis [5]. In one reported case, a cat developed a mass in the interscapular region 6 weeks after being administered a meloxicam injection [6]. It was concluded in this study, that a fast absorbing non-steroidal anti-inflammatory is unlikely to cause local inflammation, but the process of injecting a medication can lead to soft tissue inflammation and potential tumor growth [6]. We believe that this same principle could be applied when injecting a corticosteroid and could be the cause of tumor development in this case We feel that based on previous evidence in cats [6], it would be unlikely for the corticosteroid to cause local tissue inflammation, but more likely that the trauma incited by the needle penetration into the tissue would cause inflammation. In humans, it has been documented that blunt trauma or the injection of a chemical agent can lead to soft tissue injury [7]. In that case report, two patients were found to have large deep-seated masses in their thighs from injecting anabolic steroids. Histopathology revealed the masses to be reactive inflammatory responses with the presence of foreign material. These cases support the idea that local tissue reaction and the introduction of foreign material into the body is capable of causing inflammation and tumor formation, which is the basis for our hypothesis in this patient.

Because ISS are not well documented in canines, it is hard to determine what characteristics should compose this type of tumor in a dog. The tumor did possess characteristics that are commonly seen in cat ISS, such as eosinophilic cytoplasm with an abundant intercellular collagenous and myxoid matrix [6]. The nuclei were abnormally large with vesicular chromatin and prominent nucleoli, a high mitotic index and a region of necrosis was observed on histopathology. Histological features that were used to make a diagnosis of ISS in a cat include, subcutaneous location where an NSAID injection was previously given, central necrosis, giant cells, cellular pleomorphism, and myoblastic differentiation [6]. It has been reported that vaccine site sarcomas are characteristically high grade, fast growing tumors [8]. Aberdein et al reported that $30 \%$ of vaccine site sarcomas were a grade III tumor in comparison to $13 \%$ of non-vaccine site sarcomas [8]. Immunohistochemical staining for smooth muscle actin is a 
common feature of ISS [7]. In our case, the tumor was a grade III fibromyxosarcoma, but was negative for smooth muscle actin. This could indicate that the predominant cells of the tumor are fibroblasts or myofibroblasts that do not express actin [9]. This type of behavior has been previously documented in dogs with keloidal tumors [9]. Keloidal tumors in dogs have been documented to undergo malignant transformation and lead to aggressive fibrosarcomas [9]. We suspect that because our patient's tumor had similar characteristics of malignancy, it is feasible for her aggressive tumor to have developed from local inflammation secondary to an injection site.

The aim of this case report was to document the potential association between a corticosteroid injection and formation of a soft tissue tumor (injection site sarcoma). The history of the patient, aggressive nature of the tumor and location of the mass suggest a relationship between the previous corticosteroid injection and tumor development. Because this patient never received any prior injections in the limb, the corticosteroid injection may have led to tumor development in this area. There is also the possibility that the tumor growth was not associated with the injection and was previously not identified until after the injection or grew coincidentally. Previous to tumor growth, the patient had multiple corticosteroid and vaccine injections throughout her life that did not lead to tumor growth, which makes it hard to prove our hypothesis. Reports with similar aim as ours i.e. association between a non-vaccine medication injection and formation of a soft tissue tumor have been published in cats, which helps to support that although we need further information, we cannot rule out an association $[5,6,10]$. Srivastav et al documented association between long acting injectable steroids, such as methylprednisolone, dexamethasone and triamcinolone and occurrence of sarcomas in cats [5]. Other two reports documented association between development of an injection site sarcoma and meloxicam injection, and subcutaneous fluid port, with no follow up and few weeks follow up reported, respectively [6,10]. The follow up for the dog in our report was also relatively short ( 5 months) but we followed this patient until death i.e. maximum follow up possible. Due to her naturally passing at home, we cannot rule out that neoplasia was her cause of death. We do not know the association with the osteosarcoma identified on histopathology, but due to the aggressive nature of these tumors, this could be one cause for her passing [11].

\section{Conclusion}

We believe that this case is worth documenting due to the potential correlation between a corticosteroid injection and tumor development, however we cannot rule out this was an incidental finding and further cases need to be examined in order to form a causative relationship in dogs.

\section{Acknowledgement}

Authors would like to thank Stephen Joslyn, BVMS, Dip.ECVDI, MRCVS, for assistance with the three dimensional volumerendered computed tomographic images.

\section{References}

1. Woodward KN (2011) Origins of injection-site sarcomas in cats: the possible role of chronic inflammation-a review. ISRN Vet Sci 2011 : 10.5402/2011/210982.

2. Hohenhaus AE, Kelsey JL, Haddad J, Barber L, Palmisano M, et al. (2016) Canine Cutaneous and Subcutaneous Soft Tissue Sarcoma: An Evidence-Based Review of Case Management. J Am Anim Hosp Assoc 52: 77-89.

3. Vascellari M, Melchiotti E, Bozza MA, Mutinelli F (2003) Fibrosarcomas at presumed sites of injection in dogs: characteristics and comparison with nonvaccination site fibrosarcomas and feline post-vaccinal fibrosarcomas. J Vet Med A Physiol Pathol Clin Med 50: 286-91.

4. Kass PH, Spangler WL, Hendrick MJ, McGill LD, Esplin DG, et al. (2003) Multicenter case-control study of risk factors associated with development of vaccineassociated sarcomas in cats. J Am Vet Med Assoc 223: 1283-92.

5. Srivastav A, Kass PH, McGill LD, Farver TB, Kent MS (2012) Comparative vaccine-specific and other injectable-specific risks of injection-site sarcomas in cats. J Am Vet Med Assoc 241: 595-602.

6. Munday JS, Banyay K, Aberdein D, French AF (2011) Development of an injection site sarcoma shortly after meloxicam injection in an unvaccinated cat. J Feline Med Surg 13: 988-91.

7. Weinreb I, Goldblum JR, Rubin BP (2010) Factitial soft tissue pseudotumor due to injection of anabolic steroids: a report of 3 cases in 2 patients. Hum Pathol 41: 452-5.

8. Aberdein D, Munday JS, Dyer CB, Knight CG, French AF, et al. (2007) Comparison of the histology and immunohistochemistry of vaccination-site and nonvaccination-site sarcomas from cats in New Zealand. N Z Vet J 55: 203-7.

9. Mikaelian I, Gross TL (2002) Keloidal fibromas and fibrosarcomas in the dog. Vet Pathol 39: 149-53.

10. McLeland SM, Imhoff DJ, Thomas M, Powers BE, Quimby JM (2013) Subcutaneous fluid port-associated soft tissue sarcoma in a cat. J Feline Med Surg 15: 917-20.

11. Fenger JM, London CA, Kisseberth WC (2014) Canine osteosarcoma: a naturally occurring disease to inform pediatric oncology. ILAR J 55: 69-85. 


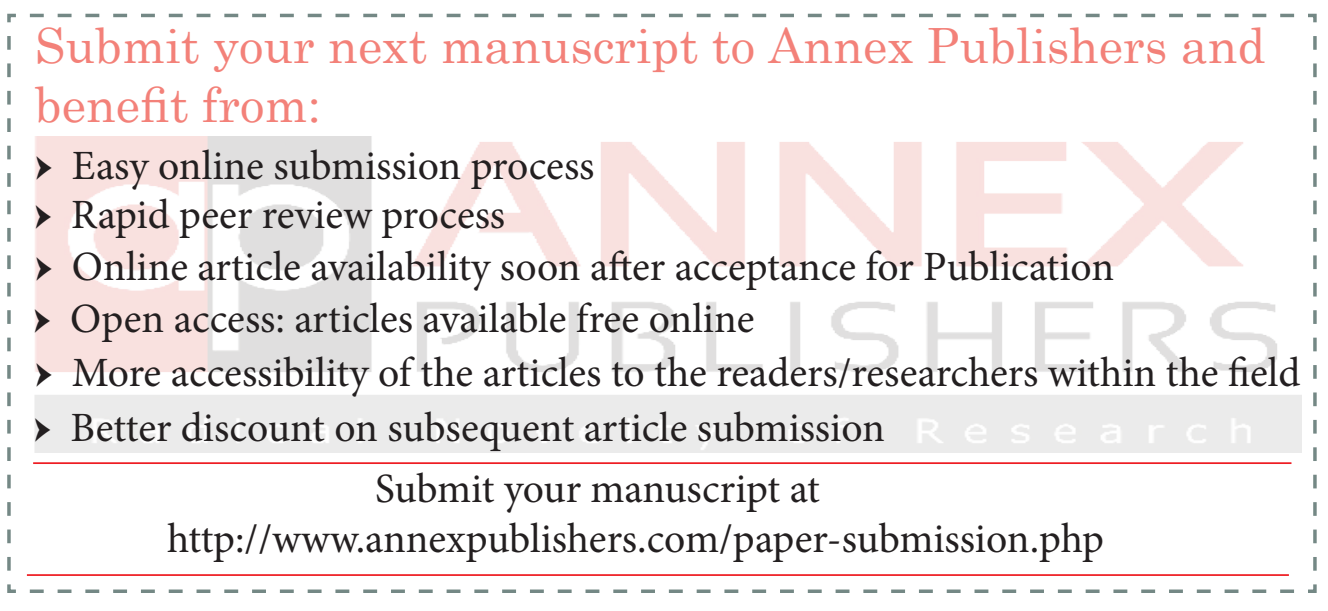

